\title{
Scaling-up Model-based Clustering Algorithm by Working on Clustering Features ${ }^{\star}$
}

\author{
Huidong Jin $^{1}$, Kwong-Sak Leung ${ }^{1}$, and Man-Leung Wong ${ }^{2}$ \\ 1 Department of Computer Sci. \& Eng., the Chinese University of Hong Kong \\ Shatin, N.T., Hong Kong. \{hdjin, ksleung\}@cse.cuhk.edu.hk \\ 2 Department of Computing and Decision Sciences, Lingnan University, \\ Tuen Mun, Hong Kong. mlwong@ln.edu.hk.
}

\begin{abstract}
In this paper, we propose EMACF (Expectation-Maximization Algorithm for Clustering Features) to generate clusters from data summaries rather than data items directly. Incorporating with an adaptive grid-based data summarization procedure, we establish a scalable clustering algorithm: gEMACF. The experimental results show that gEMACF can generate more accurate results than other scalable clustering algorithms. The experimental results also indicate that gEMACF can run two order of magnitude faster than the traditional expectation-maximization algorithm with little loss of accuracy.
\end{abstract}

\section{Introduction}

Clustering is the unsupervised classification of data items into meaningful clusters based on similarity or density. Given a data set $X=\left(\mathbf{x}_{1}, \mathbf{x}_{2}, \cdots, \mathbf{x}_{N}\right)$, the modelbased clustering algorithms assume that each data item $\mathbf{x}_{i}=\left[x_{1 i}, x_{2 i}, \cdots, x_{1 i}\right]^{T}$ is drawn from a finite mixture model $\Phi$ of $K$ distributions: $p\left(\mathbf{x}_{i} \mid \Phi\right)=\sum_{k=1}^{K} p_{k} \phi\left(\mathbf{x}_{i} \mid \theta_{k}\right)$. Here $p_{k}$ is the mixing proportion for the $k^{t h}$ cluster. In this paper, we concentrate on Gaussian mixture model. That is, the component density function $\phi\left(\mathbf{x}_{i} \mid \theta_{k}\right)$ is of the form $\phi\left(\mathbf{x}_{i} \mid \theta_{k}\right)=\frac{\exp \left\{-\frac{1}{2}\left(\mathbf{x}_{i}-\mu_{k}\right)^{T} \Sigma_{k}^{-1}\left(\mathbf{x}_{i}-\mu_{k}\right)\right\}}{(2 \pi)^{\frac{D}{2}}\left|\Sigma_{k}\right|^{\frac{1}{2}}}$ where $\mu_{k}$ is a mean vector, $\Sigma_{k}$ is a covariance matrix, $D$ is the dimensionality of the data set.

The Expectation-Maximization (EM) algorithm, an effective and popular technique for selecting mixture models, maximizes iteratively the log-likelihood $L(\Phi)=$ $\log \left[\prod_{i=1}^{N} p\left(\mathbf{x}_{i} \mid \Phi\right)\right]$. However, the EM algorithm has to read each data item many times since it reads each data item within each iteration of E-step and M-step. This prohibits it from handling large sets, especially when they are too large to fit into main memory. There are much research work to accelerate the convergence but most of them still need multiple scans of the whole data set $[3,4]$. The scalable model-based clustering algorithm in [1] only scans through a whole data set once. But it invokes its core algorithm, ExEM (Extended EM), multiple times to sum

\footnotetext{
* The work was partially supported by RGC Earmarked Grant for Research CUHK
} 4212/01E of Hong Kong. 

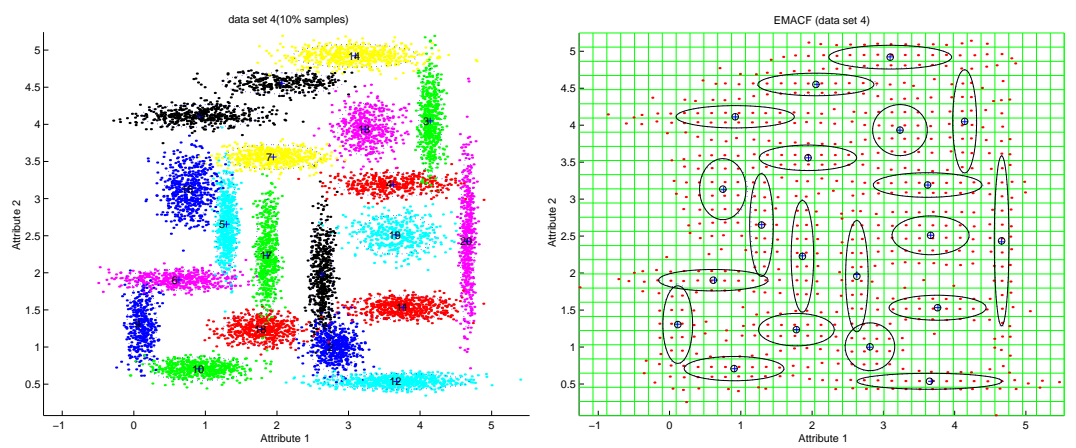

Fig. 1. (a). A data set with 100,000 data items in 20 clusters (b). the clusters generated by EMACF. A dot indicates a data item in (a) and a clustering feature in (b). The center 'o' (or ' +') and its corresponding solid (or dotted) ellipse indicate the mean and a contour of a generated (or original) component Gaussian distribution.

up the data set before generating a mixture model. Furthermore, ExEM has no convergence guarantee.

Our idea for scaling-up model-based clustering algorithms is first to partition the large data set into mutually exclusive subclusters which only contain similar data items. Each subcluster is summarized into a data summary. Then we will propose a new model-based clustering algorithm to generate clusters from data summaries directly.

\section{Adaptive data summarization procedure}

Before discussing how to summarize a data set effectively, we need to choose a proper data summary representation. Although different forms of data summaries may be used to describe a subcluster of data items $[1,4]$, they may be derived from a Clustering Feature (CF), which is originally used in [6]. In fact, under the assumption that attributes are independent in this paper, a clustering feature is a sufficient statistics of the subcluster if data items distribute normally. A clustering feature is denoted by $\mathbf{s}_{m}=\left\{n_{m}, \nu_{m}, \gamma_{m}\right\}(m=1, \ldots, M)$. Here $n_{m}$ is the cardinality of the $m^{\text {th }}$ subcluster; $\nu_{m}=\frac{1}{n_{m}} \sum_{\text {the } m^{t h} \text { subcluster }} \mathbf{x}_{i}$ is the mean; $\gamma_{m}=\left(\gamma_{1 m}, \gamma_{2 m}, \ldots\right.$, $\left.\gamma_{D m}\right)^{T}$ is the diagonal vector of the matrix $\Gamma_{m}=\frac{1}{n_{m}} \sum_{\text {the } m^{t h}} \mathbf{x}_{\text {subcluster }} \mathbf{x}_{i} \mathbf{x}_{i}^{\mathbf{T}}$, which indicates the second moment of the $m^{\text {th }}$ subcluster.

There are many approaches to summarize data sets. We employ an intuitive and simple grid-based approach. Basically, the data space is partitioned into mutually exclusive subspace and data items within same subspace form a subcluster. We use grid to partition the data space and partition each attribute into multiple segments. Thus, the data space is partitioned into many cells and data items within a same cell are quite similar to each other. Each cell may have a unique number to identify itself. To reduce the memory requirement, we only store the clustering features for these non-empty cells in a CF-array. In our implementation, 
the length of the CF-array is $\min \left(30^{D}, 3000\right)$ empirically. The index of a clustering feature is determined by a hash function based on its cell number. When a new data item comes, starting from the hash index, we look for an entry in the CFarray to assimilate the data item by updating the clustering feature. This entry may be empty or already be occupied by the associated cell. When the CF-array is full, the smallest width of cells is doubled, and pairs of neighboring cells are merged into bigger ones to release some memory for forthcoming data items. The cell width may increase gradually to make better use of main memory. In addition, when it reconstructs a new CF-array, only the clustering features in the old CFarray are involved. Therefore, the procedure merely scans through the whole data sets once. For the data set shown in Fig. 1 (a), the procedure can generate 656 non-empty clustering features as plotted in Fig. 1(b).

\section{An EM Algorithm for Clustering Features}

For each subcluster, the second moment $\gamma_{m}$ may represent data dispersion within a subcluster. Taking account of the second moment directly in the clustering algorithms, we may generate more accurate results. On the other hand, the Gaussian mixture models contain the second moment too. This enables us to embody entire clustering features explicitly in our new algorithm.

For each data item $\mathbf{x}_{i}$ in the $m^{\text {th }}$ subcluster, we give a 'density function' to approximate the normal distribution, specified as follows:

$\psi\left(\mathbf{x}_{i} \in m^{t h}\right.$ subcluster $\left.\mid \theta_{k}\right) \triangleq \psi\left(\mathbf{s}_{m} \mid \theta_{k}\right)=\prod_{d=1}^{D} \frac{\exp \left\{-\frac{1}{2 \sigma_{d k}}\left(\gamma_{d m}-2 \mu_{d k} \nu_{d m}+\mu_{d k}^{2}\right)\right\}}{(2 \pi)^{\frac{1}{2}} \sigma_{d k}^{\frac{1}{2}}}$.

It is a normal density function when $\gamma_{d m}=\nu_{d m}^{2}$. With this function, the probability of a data item $\mathbf{x}_{i}$ explicitly depends on which subcluster it belongs to. It is only implicitly relevant with its values. The function enables us to treat data items in a subcluster in the same way, so we can just store the clustering features to reduce lots of memory usage as well as execution time. In addition, intuitively, if the second moment $\gamma_{m}$ is small, that is, data items are quite dense, the subcluster has more influence on clustering. This accords with the model-based clustering principle to locate clusters in dense regions.

With this density function, the probability for a data item $\mathbf{x}_{i}$ in the $m^{t h}$ subcluster under the mixture model is $p\left(\mathbf{x}_{i} \in\right.$ the $m^{t h}$ subcluster $\left.\mid \Psi\right) \triangleq p\left(\mathbf{s}_{m} \mid \Psi\right)=$ $\sum_{k=1}^{K} p_{k} \psi\left(\mathbf{s}_{m} \mid \mu_{k}, \sigma_{k}\right)$. Then the log-likelihood is given as $L(\Psi)=\sum_{m=1}^{M} n_{m} \log p\left(\mathbf{s}_{m} \mid \Psi\right)$. Based on these definitions, we derive the EM algorithm for Clustering Features (EMACF) based on the general EM algorithm [3].

1. E-step: Given the mixture model parameters $\Psi^{(j)}$, compute the membership $r_{m k}^{(j)}$ for each subcluster:

$$
r_{m k}^{(j)}=\frac{p_{k}^{(j)} \psi\left(\mathbf{s}_{m} \mid u_{k}^{(j)}, \sigma_{k}^{(j)}\right)}{\sum_{i=1}^{K} p_{i}^{(j)} \psi\left(\mathbf{s}_{m} \mid u_{k}^{(j)}, \sigma_{k}^{(j)}\right)}
$$


Table 1. The clustering accuracy of 6 clustering algorithms on 10 data sets.

\begin{tabular}{|c|r|r|r|l|l|l|l|l|l|}
\hline Data Set & \multicolumn{1}{|c|}{$N$} & $D$ & $K$ & gEMACF & gExEM & gEMAWS & iEM & sampiEM & IBS \\
\hline 1 & 60000 & 2 & 6 & $\mathbf{0 . 9 6 2}$ & 0.934 & 0.942 & 0.960 & 0.958 & 0.836 \\
\hline 2 & 480000 & 2 & 16 & $\mathbf{0 . 8 8 5}$ & 0.882 & 0.819 & 0.855 & 0.827 & 0.671 \\
\hline 3 & 100000 & 2 & 9 & $\mathbf{0 . 7 8 5}$ & 0.676 & 0.782 & 0.748 & 0.745 & 0.553 \\
\hline 4 & 100000 & 2 & 20 & 0.851 & 0.847 & 0.739 & $\mathbf{0 . 8 5 8}$ & 0.846 & 0.632 \\
\hline 5 & 120000 & 2 & 31 & 0.893 & 0.854 & 0.819 & $\mathbf{0 . 9 0 5}$ & 0.875 & 0.697 \\
\hline 6 & 120000 & 2 & 41 & $\mathbf{0 . 8 7 3}$ & 0.865 & 0.727 & 0.871 & 0.839 & 0.657 \\
\hline 7 & 100000 & 3 & 10 & 0.911 & $\mathbf{0 . 9 1 7}$ & 0.854 & 0.892 & 0.770 & 0.676 \\
\hline 8 & 100000 & 4 & 10 & $\mathbf{0 . 9 3 1}$ & 0.927 & 0.920 & 0.930 & 0.925 & 0.825 \\
\hline 9 & 100000 & 5 & 10 & $\mathbf{0 . 8 6 8}$ & 0.816 & 0.820 & 0.856 & 0.843 & 0.741 \\
\hline 10 & 100000 & 6 & 10 & $\mathbf{0 . 9 3 4}$ & $\mathbf{0 . 9 3 4}$ & 0.800 & 0.904 & 0.896 & 0.690 \\
\hline Average & \multicolumn{10}{|c|}{} & & $\mathbf{0 . 8 8 9}$ & 0.865 & 0.822 & 0.878 & 0.853 & 0.698 \\
\hline
\end{tabular}

2. M-step: Given $r_{m k}^{(j)}$, update the mixture model parameters for $k=1, \ldots, K$ :

$$
\begin{aligned}
p_{k}^{(j+1)} & =\frac{1}{N} \sum_{m=1}^{M} n_{m} r_{m k}^{(j)}, \\
\mu_{k}^{(j+1)} & =\frac{\sum_{i=1}^{M} n_{m} r_{m k}^{(j)} \nu_{m}^{(j)}}{\sum_{m=1}^{M} n_{m} r_{m k}^{(j)}}=\frac{\sum_{i=1}^{M} n_{m} r_{m k}^{(j)} \nu_{m}^{(j)}}{N \cdot p_{k}^{(j+1)}}, \\
\sigma_{k}^{(j+1)} & =\frac{\sum_{m=1}^{M} n_{m} r_{m k}^{(j)}\left[\gamma_{m}-2 \mu_{k}^{(j)} \circledast \nu_{m}+\mu_{k}^{(j)} \circledast \mu_{k}^{(j)}\right]}{N \cdot p_{k}^{(j+1)}}
\end{aligned}
$$

where $\circledast$ indicates the array multiplication.

The algorithm ends when the log-likelihood values $L\left(\Psi^{(j)}\right)$ changes little. This criterion is certainly reachable as supported by the convergence theorem [2]. It is easy to see that the computation complexity of EMACF is $O(M K D)$ if the loop is terminated after perdefined number of iterations.

\section{Experiments}

Now, we compare our proposed algorithm with other scalable clustering algorithms. Working on the clustering features generated by the adaptive grid-based data summarization procedure, EMACF may form a scalable clustering algorithm, identified as gEMACF below. If the second moment $\gamma_{m}$ in EMACF is replaced by $v_{m} \circledast v_{m}$, EMACF is simplied into an EM Algorithm for Weighted Samples (EMAWS). That scalable algorithm is denoted by gEMAWS. Similarly, if the ExEM algorithm works on the clustering features [1], we can construct a scalable algorithm: gExEM. We also compare gEMACF with the Inverse Biased Sampling (IBS) algorithm. It is a typical implementation of density-biased sampling algorithm, which outperforms BIRCH on skewed data sets [5]. To highlight the 


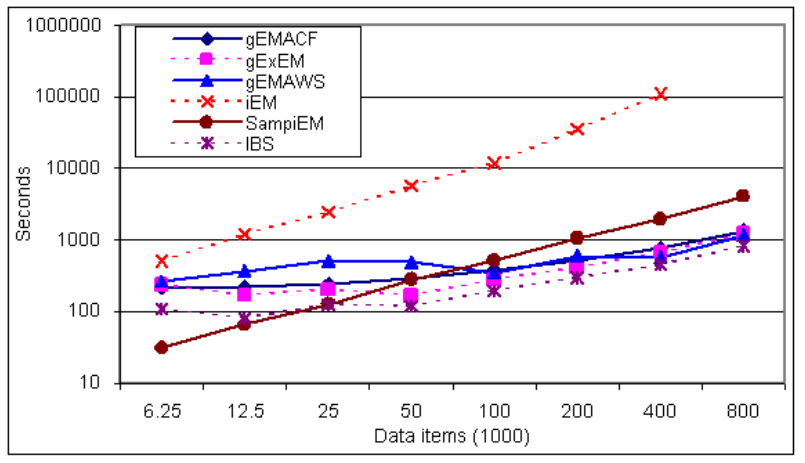

Fig. 2. Execution time on 8 4-dimensional data sets.

scalability of these algorithms, we also compare them with the traditional EM algorithm. It is denoted by iEM, where i indicates that attributes are independent as in EMACF and ExEM. SampiEM stands for iEM working on 5\% random samples. We include it because sampling is a common strategy for scaling-up data mining algorithms [1,5]. All algorithms were implemented with MATLAB and ran on a Sun Enterprise E4500. The results were averaged on 10 runs.

Table 1 illustrates the clustering accuracy of these 6 algorithms on 10 data sets. These data sets are generated according to 10 random mixture models whose parameters are given in columns 2,3 , and 4 . The data size ranges from 6,400 to 480,000 and their dimensionality ranges from 2 to 6 . Fig. 1(a) illustrates the fourth data set. The clustering accuracy indicates the proportion of data items that are correctly clustered with respect to the original mixture models. Observed from the table, gEMACF generates better clustering results than the others do except data sets 4, 5 and 7 where gEMACF performs sightly worse. Fig.1(b) illustrates a mixture model generated by gEMACF which well describes the data set in Fig.1(a). The clustering accuracy of gEMACF ranges from $78.5 \%$ to $96.2 \%$. On average, the clustering accuracy of gEMACF, gExEM, gEMAWS, SampiEM and iEM algorithm are $88.9 \%, 86.5 \%, 82.2 \%, 85.3 \%$ and $87.8 \%$ respectively. The gEMACF algorithm can generate more accurate results. It is interesting to see that gEMACF performs even slightly better than the iEM algorithm. This partially because that the smaller number of clustering features may cause smaller number of local maxima in the loglikelihood space. The gEMACF algorithm performs much better than SampiEM because sampling usually introduces some biases. The average clustering accuracy for the distance-based clustering algorithms, IBS, is much worse than that of the model-based clustering algorithms. Because all mixture models involved are so skew that they are not suitable for the distance-based clustering algorithm.

Fig.2 illustrates the execution time for the 6 clustering algorithms on 8 4dimensional data sets. All data sets have 10 clusters. Observed from the figure, the execution time of gEMACF, gExEM, gEMAWS and IBS increases slowly while iEM and SampiEM increases more quickly with the size of data set. The first 4 algorithms take similar execution time and all scale up well with large data 
sets. Because the data summarization procedure takes longer time than sampling for the first 3 small data sets, the first four algorithms execute slightly slower than SampiEM. However, for the last three large data sets, the difference between gEMACF and SampiEM/iEM becomes quite significant. For example, gEMACF only takes about 1344 seconds for the largest data set with 800,000 data items. It runs 3 times faster than SampiEM which needs 4050 seconds. Moreover, the traditional algorithm, iEM, failed to generate mixture models for the data set after executing for two days. Even for the data set with 400,000 data times, iEM needs about 110,245 seconds to generate a mixture model for the data set. The gEMACF algorithm only takes about 775 seconds which is about 142.7 times faster than iEM. For these data sets, the smallest speedup of gEMACF is obtained for the smallest data set with a value of 2.4. Thus, gEMACF is much faster than the traditional EM algorithm.

\section{Conclusion}

The EMACF (Expectation-Maximization Algorithm for Clustering Features) algorithm has been proposed to make better use of clustering features. It guarantees to converge. We have examined its performance by incorporating it with an adaptive grid-based data summarization procedure to construct a scalable algorithm: gEMACF. The experimental results have shown that the gEMACF algorithm can run two order of magnitude faster than the traditional EM algorithm. It can generate clustering results more accurate than other scalable model-based clustering algorithms with similar computation resources. It is subject to our future research on integrating with other data summarization procedure to establish scalable clustering algorithms for high-dimensional data sets. Another interesting direction of this work is to establish other model-based clustering algorithms on data summaries of complicated data sets.

\section{References}

1. P. Bradley, U. Fayyad, and C.R. Reina. Clustering very large databases using EM mixture models. In Proceedings of 15th International Conference on Pattern Recognition, volume 2, pages 76-80, 2000.

2. Huidong Jin, Kwong-Sak Leung, and Man-Leung Wong. An expectation-maximization algorithm working on data summary. In Second International Workshop on Intelligent Systems Design and Applications, Mar. 2002 (Accepted).

3. G. J. McLachlan and T. Krishnan. The EM Algorithm and Extensions. John Wiley \& Sons, Inc., New York, 1997.

4. A. Moore. Very fast EM-based mixture model clustering using multiresolution KDtrees. In Advances in Neural Information Processing Systems 11, pages 543-549, 1999.

5. Christopher R. Palmer and Christos Faloutsos. Density biased sampling: An improved method for data mining and clustering. In Proceedings of the 2000 ACM SIGMOD international conference on Management of data, pages 82-92, 2000.

6. Tian Zhang, Raghu Ramakrishnan, and Miron Livny. BIRCH: an efficient data clustering method for very large databases. In Proceedings of the 1996 ACM SIGMOD international conference on Management of data, pages 103-114, 1996. 